Classification

Physics Abstracts

$07.80-29.30-82.80$

\title{
The impact of EELS in materials science
}

\author{
Christian Colliex
}

Laboratoire de Physique des Solides, Université Paris-Sud, Bâtiment 510, 91405 Orsay Cedex, France

(Received April 05, 1991; accepted April 12, 1991)

\begin{abstract}
This summary paper emphasizes the highlights of the workshop which are particularly relevant for applications in materials science. It reviews progress in EELS instrumentation, data recording and processing, and in the use and interpretation of EELS fine structures. We point out the possibilities of EELS as a local analytical tool, discuss EELS detection limits, and indicate potential fields of applications for EELS fine structure studies.
\end{abstract}

\section{Introduction.}

An EELS measurement concerns a scattering event, in which a primary electron transfers momentum and energy to a solid target. This is the type of experiment that physicists like to work on. As a result, new sources, spectrometers and detectors are being unceasingly developed to record more accurate data. The physicists then use the data to determine various fundamental properties of well defined specimens, such as scattering cross-sections, dispersion curves, plasmon energies and lifetimes. This type of activity is a prerequisite for establishing EELS as a useful tool in solid specimen characterization. However, it has made much of the materials science community feel that: "EELS is a playground for physicists and is of little help for solving materials science problems". This paper attempts to show that even though EELS requires more complex routines of data processing and thinner specimens than energy-dispersive X-ray spectroscopy (EDX), the rich variety of obtainable information make EELS into an indispensable materials science tool.

When exploring the potential contribution of EELS to materials science, it is useful to review the type of problems raised by real materials. In contrast with the ideal specimens of the physics world, real materials have to possess some unique properties of interest for microelectronics, chemistry, aerospace, etc., i.e. for industries with a productive potential. New processing methods create materials with more complex structures and inhomogeneities on shorter dimensions: multilayered components, nanostructured crystals. When studying such materials, one aims to determine the local microstructure, local elemental composition and eventually local bonding effects. This "local" character, which was at the micron level ten years ago or more, is nowadays clearly required at the nanometer level. Probably the most important aspect of EELS in materials science is that it constitutes one of the very few techniques capable of providing such informations at this scale. 
During the course of the workshop, recent substantial advances in instrumentation as well as in data processing were presented, and various current projects were discussed. In the following, I shall summarize some general trends which appeared, and I shall comment on their potential applications for solving materials science problems.

\section{EELS instrumentation.}

2.1 PRESENT PERFORMANCE. - In the point analysis mode, the magnetic spectrometer designed with second-order aberration correction offers an energy resolution of $0.3-0.5 \mathrm{eV}$ when operated on a column equipped with a cold field emission gun, and $1-1.5 \mathrm{eV}$ with a $\mathrm{LaB}_{6}$ filament. An improved energy resolution of the order of $0.1 \mathrm{eV}$ has been demonstrated on FEG-STEM equipped with a Wien filter (Batson [1]). This performance is very competitive with synchrotron radiation for core level spectroscopy in the $100-1000 \mathrm{eV}$ range.

When investigating the spatial distribution of an EELS feature, two approaches are available: the STEM mode with an EELS spectrometer, and the energy filtering TEM (EFTEM). In the first case, one or several channels in the EELS spectrum are acquired for each probe position and one or several energy filtered images result from scanning the probe across the specimen. This mode has been used for elemental mapping of clusters and interfaces with subnanometer resolution of the incident probe (Colliex et al. [2], Mory and Colliex [3]) In the EFTEM case, a 2D image is collected for each energy window at a time. The prototype of this filtering device, incorporated in the mid of a TEM column, is the Castaing-Henry design, commercially updated by Zeiss. More recent versions, known as $\Omega$-filters, are made of a combination of 4 magnetic sectors. The advantages and deficiencies of the STEM and EFTEM approaches have often been discussed - see for instance Leapman and Andrews [4].

The major progress over the last few years has been the realization of PEELS of practical use (see for instance Krivanek et al. [5]). Most experimental results presented at the workshop have been recorded with such devices, which are much more efficient and user-friendly than previous models. It is now routinely possible to record a spectrum for quantitative elemental analysis within one or two seconds. The acquisition of a detailed edge profile for fine structure analysis is typically a matter of 10 seconds.

2.2 USEFUL DEVELOPMENTS TO BE ANTICIPATED. - In the continuation of the technology presently available, different proposals and realisations have been reported during the workshop and they can be used to outline the future of EELS instrumentation.

Some of them concern the improvement of energy resolution. Even though the currently resolution is not far from the ultimate limit for core-hole spectroscopy imposed by core hole lifetimes, it is not sufficient for investigations of band structures, energy levels of electronic defects and of vibrational modes. To improve the energy resolution to a few $\mathrm{meV}$, it is necessary to monochromate the electron beam before it reaches the specimen (see Krivanek et al. [6], Terauchi et al. [7]).

As for spatially-resolved EELS, both the STEM and EFTEM approaches are benefiting from improvements in optics and detector design. For instance, in the VG-STEM, an objective lens of low $C_{\mathrm{s}}$ is now available, delivering a $0.2-0.3 \mathrm{~nm}$ probe diameter on the specimen. Fast parallel detection with single electron counting capability and quick read-out of the spectrum is presently under development [6] in order to satisfy the requirements for elemental mapping with core losses. This constitutes the best solution in terms of signal acquisition efficiency for recording spectrumimages, such as proposed by Jeanguillaume and Colliex [8]. For the EFTEM approach, alternative solutions requiring only one magnetic sector at the bottom of the EM column have been proposed 
[9]. During the workshop, Krivanek has introduced a new imaging spectrometer in which the post-magnet optics which produces energy filtered images has been developed to a high degree of refinement, and now includes six quadrupole and six sextupole lenses in front of a 2D slow scan CCD camera [6].

Whichever mode, STEM or EFTEM, is used, energy filtered images with atomic resolution will soon be available. Consequently, chemical mapping, atomic layer per atomic layer, or atomic column per atomic column, lies within the realm of feasibility.

\section{EELS as a microanalytical tool.}

In materials science, EELS has mostly been recognized as a tool for quantitative elemental analysis. Over the last decade the scheme used for EELS quantitation is that proposed and worked out by Egerton in the late seventies [10]. The elemental signal is measured after background modelling and subtraction, and the elemental concentration is estimated from a linear relationship which relates the measured signal to the number of atoms through a weighting factor known as the partial cross section. The use of this method has been extended to moderatly thick specimens by introducing appropriate deconvolution techniques with the low loss spectrum.

Two recently introduced spectrum processing techniques have the potential to substantially change the attitude of materials scientists to EELS. Let us identify these new developments.

3.1 SignAL DETECTION FOR LOW CONCENTRATIONS. - The detection of the EELS signal due to a weakly present element is difficult, and the standard approach of background extrapolation cannot be followed. This is especially true when the edge is so weak that the signal-to-background ratio $S / B \lesssim 0.001$. It is also true when the background cannot be modelled with a simple power law, as happens for the case of overlapping edges and of background oscillations due to extended fine structures. Moreover, the use of parallel detectors introduces an extra source of systematic noise, known as the channel-to-channel gain variation. These considerations have stimulated the development of more suitable methods for edge identification using first - or second-difference acquisition schemes. The ultimate level is the automatic EELS feature detection described in these proceedings (Kundmann and Krivanek [11]).

3.2 SIGNAL EXTRACTION AND QUANTIFICATION. - Spectral processing algorithms suitable for the quantification of the EELS signal from weakly present elements, which are rather similar to those used in EDX, are now being introduced. The principle is to fit the experimental spectrum with a model constructed as a sum consisting of a background and of core edges [12-14]. Whereas the EDX lines all have a similar shape governed by the poor energy resolution of the detector, EELS edges are more complex, they extend over large energy windows, and their detailed profiles depend on the specimen itself. Moreover, higher energy plasmon satellites tend to appear in thicker specimens. All these features must be taken into account and require a refined analysis for a reliable use in quantitation.

In any quantification scheme, the important parameter is the differential cross section $\frac{\mathrm{d} \sigma}{\mathrm{d} E}(E)$ of the edge, calculated or measured over a substantial energy range above the edge threshold. There exist two ways for improving our knowledge of cross sections and we advocate developing both of them:

- The first solution is to use reference edges recorded on standard specimens. This method was introduced by Shuman and Somlyo [12] in their work on low concentrations of $\mathrm{Ca}$ in a carbonaceous matrix. The method is valid when there are no substantial changes between the 
edge shapes in the unknown material and in the reference specimen. However, in materials science, noticeable changes in fine structures can be induced by variations in local bonding and environment. Consequently, it is recommended to store as many as possible differential cross sections obtained from standards. This calls for a second generation of the EELS atlas [15], which would provide the community of EELS users with an extended data library available in a convenient computer format.

- The second solution is to deepen our theoretical knowledge of cross-sections. As a specific example, let us point out the current situation with $\mathrm{L}_{23}$ and $\mathrm{M}_{45}$ edges: the white line contribution must be removed from the experimental spectrum before comparison with Hartree-Slater calculations, which do not consider transitions towards bound states [16], even though it constitutes the major feature available on these edges.

Once these two developements have been realized, it not unreasonable to expect an improvement in the accuracy of the absolute elemental quantification from an average of $10 \%$ down to a few atomic \%.

3.3 DETECTION LIMITS. - Before undertaking any microanalytical experiment with EELS (this is also true for EDX or any other analytical technique), it is most important to evaluate the range of accessible performance. Two types of detection limits have been defined and evaluated by Isaacson and Jonhson [17]: the minimum detectable mass (MDM) or minimum detectable number of atoms (MDN), and the minimum detectable mass fraction (MMF). The first limit applies in situations in which an element $B$ is segregated in matrix $A$, while the second one applies to a homogeneous distribution of B within A.

It may be useful to provide a few guidelines, so that materials scientists can evaluate a priori the chance of success for a given specimen and a given instrument. The important parameters are as follows:

- specimen thickness: $t$ (nm);

- incident probe diameter: $d(\mathrm{~nm})$ so that the irradiated area is $\simeq d^{2}$.

- analysed volume $\simeq t d^{2}$

- total number of atoms in the analysed volume: $N \simeq n t d^{2}$ where $n$ is the average number of atoms per unit volume;

- the specimen consists of a concentration $C$ of impurity atoms B within a matrix made of atoms A (generally $C<<1)$;

- number of impurity atoms B in the analysed volume:

$$
N_{\mathrm{B}}=C n t d^{2}
$$

- total probe current: $I\left(\mathrm{e}^{-} / \mathrm{s}\right)$;

- probe current density: $J=I / d^{2}$;

- irradiation dose fore the experiment: $D=J T$;

- recording time: $\tau$;

- ratio of recording time to irradiation time: $\tau / T(\tau / T=1$ for PEELS and $\simeq 0.01$ for serial EELS);

- core-edge signal $\mathrm{K}$ used for the analysis with $S / B$, the peak/background ratio, and the probability $P$ of core-edge excitation in a foil of pure element B and thickness $t\left(P=\sigma_{\mathrm{K}} n t\right.$ where $\sigma_{\mathrm{K}}$ is the core-edge cross section).

The attainable performance is best plotted on a concentration $v$ s. resolution graph $[2,18,19]$ shown in figure 1. The term "resolution" is slightly inaccurate, in the sense that we equate "resolution" to the dimension $d$ of the incident probe. This implies that we neglect effects such as beam spreading in the foil and the non-local character of the interaction. We are also well aware that 
"resolution" must not be confused with the size of the smallest detectable objet [20]. However our simplified approach has the advantage of convenience, and provides satisfactory orders-ofmagnitude estimates when the analysis is performed at moderate resolution ( $\gtrsim 1 \mathrm{~nm}$ ) relative to the dimension of an atom. Moreover, it is useful in identifying the optimal experimental conditions for each practical experiment. The basic equation relating the measurable concentration to the signal to noise ratio (SNR) is:

$$
C=\frac{\mathrm{SNR}}{d} \cdot \frac{1}{\left[\frac{S}{B} \cdot P \cdot D \frac{\tau}{T}\right]^{1 / 2}}
$$

(see Colliex et al. [2] for the derivation of this equation). One can deduce simple consequences from this equation:

- The minimum concentration $C_{\min }$ corresponding to $\mathrm{SNR} \simeq 3$ requires a primary number of electrons $d \cdot D^{1 / 2}$ such that:

$$
C_{\min } \alpha \frac{1}{d \cdot D^{1 / 2}} \alpha \frac{1}{(I \cdot \tau)^{1 / 2}} \text { or } \frac{1}{(I \cdot T)^{1 / 2}} \text { for PEELS }
$$

This means that the smallest concentration detectable within a given time depends only on the total intensity of the electron probe. When searching for small concentrations of elements homogeneously spread throughout the specimen, it is therefore recommended to use large and intense probes.

- In contrast, the minimum detectable number of atoms is $N_{\min }=C_{\min } n t d^{2}$. It varies as:

$$
N_{\min } \alpha \frac{t}{(J T)^{1 / 2}} \text { for PEELS. }
$$

Making $N_{\min }$ as low as possible requires the thinnest specimens and the highest probe current density, attainable only with a high-brightness electron source, such as field emission gun. This is the recommended configuration for exploring highly segregated assemblies of impurities, for instance along an interface, or on a dislocation core.

The concentration $v s$ resolution graph in figure 1 is parametrized with different families of lines:

- lines of constant number of detectable atoms (with slope $=-2$ ), corresponding to $N_{\min }$ (these have been calculated for a specimen thickness $t=50 \mathrm{~nm})$;

- lines of constant incident dose $D$ and SNR (with slope $=-1$ ). They have been calculated with $\frac{S}{B}=1$ and $\sigma_{\mathrm{K}}=10^{-7} \mathrm{~nm}^{2}$, i.e. $P=5 \times 10^{-4}\left(n=100\right.$ atoms $\left./ \mathrm{nm}^{3}\right)$.

We have also plotted two specific curves corresponding to the performance attainable in $1 \mathrm{~s}$ with a PEELS device on a VG STEM equipped with a field emission source, and on an Akashi $002 \mathrm{~B}$ TEM equipped with an $\mathrm{LaB}_{6}$ filament. They take into account the relation between intensity and probe size for each instrument respectively.

The graph shows that minimum concentrations of $10^{-4}$ at. and below should be detectable with high probes of high total current (typically $100 \mathrm{nA}$ in $100 \mathrm{~nm}$ ). Single atom detection requires probes of high brightness (typically a few tenths of $\mathrm{nA}$ in a probe of a few Angströms) and thin specimens. It can only be performed with a field emission gun instrument, as demonstrated by Bouchet et al. [21]. 




Fig. 1. - Definition and estimation on a (concentration, resolution) graph of the important parameters involved in detection limits: spatial resolution $(d)$, minimum concentration $\left(C_{\min }\right)$, minimum number of atoms $\left(N_{\min }\right)$, dose $(D)$. Two typical examples of performance accessible for a 1 second PEELS measurement with a VG-HB501 FEG STEM and an Akashi 002B LaB 6 TEM/STEM are indicated for illustration. The numbers between brackets along these two curves refer to typical beam currents within probes of such diameters. The numerical values calculated on this graph correspond to specific conditions in terms of specimen thickness, signal cross-section, etc. They cannot be used straighforwardly for other experimental situations. For instance, when one aims at single atom identification with a VG microscope, the thickness of the carbon supporting layer is only of the order of $5 \mathrm{~nm}$, so that the lines of constant number of detectable atoms have to be shifted upwards by an order of magnitude. Furthermore, the dose has to be changed because the rate $P$ of the useful signal is reduced by a factor of 10 , so that the indicated curve for 1 second (VG) should then be labeled as 10 seconds (VG).

\section{EELS fine structures.}

Another highlight for the future of EELS in materials science was clearly emphasized during the workshop: it concerns EELS fine structures, more particularly those at threshold or in the ELNES domain, i.e. within typically 20 to $40 \mathrm{eV}$ above the ionization edge threshold.

It was noticed already a long time ago that these fine structures are related to electronic and 
structural parameters on the site of the excited orbital (density of states, charge transfer, symmetry of the environment...). During the workshop, great progress was demonstrated in classifying and interpreting the observed bchaviours of fine structures. Several developments were due to theoricists working on X-ray absorption spectroscopy (XAS) data delivered by synchrotron sources. One of the results of the workshop has been an increased mutual awareness of the XAS and EELS communities, recently also advocated by Sawatzky [22]: "it is of importance to properly utilize the knowledge and expertise available by including XAS in EELS conferences and, to some extent, vice versa". If the transfer of information from XAS to EELS people was evident at the workshop through the contributions of Sawatzly [23] and Stöhr, it is hoped that the reverse will occur during one of the future XAS conferences!!

Theoretical approaches in terms of molecular orbitals $[23,24]$ and of projected density of states [25] were presented at the workshop along with their successes for the interpretation of spectral features. Perhaps the most promising direction in the near future lies in the "fingerprint" information contained in the ELNES structures as analysed by Brydson et al. [24] who have shown how much the structures are governed by the arrangement and type of atoms in the first coordination shell for a wide range of materials. This methodology mostly relies on the availability of a large catalogue of edges - here is another strong argument for an extended version of the EELS atlas - and it also promotes molecular orbital calculations of larger clusters around the excited atom. Despite its difficulties, the fingerprinting approach currently seems to provide the most useful practical tool for unraveling slight changes in local coordination within complex specimens. Various examples were given by Brydson $e$ al., such as the determination of the local site symmetry of $\mathrm{Al}$ and $\mathrm{Be}$ in minerals - chrysoberyl and rhodizite [26]; the use of the $\mathrm{Ti} \mathrm{L}_{23}$ edge fine structure for discriminating between rutile and anatase [27]; the evidence for trigonal coordination of boron to oxygen in a boron-doped Fe-Cr-Mn oxide film [28], etc.

Another important source of information lies in the well-known white lines which appear on transition metal $\mathrm{L}_{23}$ and rare-earth $\mathrm{M}_{45}$ edges; they are caused by the intense transition rates of $2 \mathrm{p} \rightarrow 3 \mathrm{~d}$ bound states and $3 \mathrm{~d} \rightarrow 4 \mathrm{f}$, respectively. The relevant theory has been worked out by Fuggle, Sawatzky, and coworkers - see [23] for a review - using an atomic multiplet description with inclusion of the cubic crystal field. Practical applications in materials science include the measurement of the total weight of the $\mathrm{L}_{23}$ line compared to the weight of the transitions to continuum vacant states, and showing how the ratio is connected to d-band occupancy. It has been helpful in the understanding of the electronic structure of $\mathrm{Cu}, \mathrm{Ni}$, and $\mathrm{Fe}$ alloys and their phase transitions [29]. In a recent study, the sensitivity of the $\mathrm{Cu}-\mathrm{L}_{23}$ white line intensity to the nature of short range order in Cu-Pd alloys has been demonstrated [30]. In other cases, one is more interested in the changes in the ratio of the white-line intensities in 3d-transition metals and oxides. There exists a clear relationship between the behaviour of the ratio and the local magnetic moment $[31,32]$, so that the EELS fine structures can also provide an information about the magnetic properties of the specimen - see for instance Kurata et Tanaka [33].

The examples given above show the wealth of potential information obtained by core-loss spectroscopy. It should, be stressed again that unlike XAS, the EELS fine structure studies can be performed on nanometer-size volumes of materials. The list of promising fields of applications in materials science is very long. It includes bonding effects in homo- or heteroboundaries, electronic structures of defects, coupling of magnetic moments in magnetic ultrafine layers and multilayers, etc. There is no doubt that this type of use of EELS will be of growing importance during the next few years. 


\section{Conclusion.}

The diversity of information that can be extracted from materials science specimens by EELS spectroscopy has been clearly demonstrated, even without including the information contained in valence loss spectra as described by Howie [34]. The most important aspect of EELS is the spatially resolved character of the recorded information. In special cases, the combination of field emission electron sources with PEELS devices permits the identification of atomic size objects and the recording of fine structures from individual defects [21]. Furthermore, the rapidity of EELS data acquisition also opens the way to time-resolved studies, on a millisecond time scale for the time-evolution of gross features in the spectra, and on a scale of seconds for fine structures. In-situ studies, under the influence of an external parameter (temperature change, irradiation with photons or charged particles, deformation), constitute another wide domain for future applications for EELS in materials science.

The rich possibilities of EELS raise interesting fundamental questions when one intends to link the results with macroscopic properties. These include the sampling problem and the resultant requirement of numerous measurements, the importance of the boundary conditions due to the presence of free surfaces close to the analysed volume, the validity of models used for data interpretation. Further, how valid is it to use macroscopic quantities, such as optical or conductivity coefficients, for the description of nanometer-sized objects? These are questions open for the future.

\section{Acknowledgements.}

Thanks are due to Gatan Inc. for their support which offered me the possibility of attending the very stimulating workshop, and to O.L. Krivanek for useful comments during the preparation of this text.

References

[1] BATSON P., Microsc. Microanal. Microstruct. 2 (1991) 395.

[2] COlliex C., MaURICE J.L., UgaRTE D., Ultramicroscopy 29 (1989) 31.

[3] MORY C., COLlIEX C., Ultramicroscopy 28 (1989) 339.

[4] LEAPMAN R.D., ANDREWS S.B., Microsc. Microanal Microstruct. 2 (1991) 387.

[5] KRIVANEK O.L., AHN C.C., KEENEY R.B., Ultramicroscopy 22 (1987) 103.

[6] KRIVANeK O.L., GubBens A.J., Dellby N., Microsc. Microanal. Microstruct. 2 (1991) 315.

[7] Terauchi M., KuZuo R., Satoh F., Tanaka M., Tsuno K., Ohyama J., Microsc. Microanal. Microstruct. 2 (1991) 351.

[8] JeAnguillaume C., Colliex C., Ultramicroscopy 28 (1989) 252.

[9] Shuman H., Somlyo A.P., in Analytical Electron Microscopy 1981, R.H. Geiss Ed. (San Francisco Press, San Francisco) 202.

[10] EGERTON R.F., Electron Energy Loss Spectroscopy in the Electron Microscope (Plenum Press, 1986).

[11] Kundman M.K., KRIVANEK O.L., Microsc. Microanal. Microstruct. 2 (1991) 245.

[12] SHUMAN H., SOMLYO A.P., Ultramicroscopy 21 (1987) 23.

[13] LEAPMAN R.D., SWYT C.R., Ultramicroscopy 26 (1988) 343.

[14] Manoubi T., Tencé M., Walls M.G., CollieX C., Microsc. Microanal. Microstruct. 1 (1991) 23.

[15] AHN C.C., KRIVANEK O.L., EELS Atlas (1983), available from Gatan Inc. or HREM Facility, ASU, Tempe (U.S.A.).

[16] Manoubi T., ReZ P., Colliex C., J. Elect. Spectr. Relat. Phenom. 50 (1990) 1. 
[17] ISAACSON M., JOHNSON D., Ultramicroscopy 1 (1975) 33.

[18] CASTAING R., Analusis 15 (1987) 517.

[19] CaZAUX J., J. Microscopy 145 (1987) 257.

[20] CazauX J., COlliex C., J. Elect. Spectr. Relat. Phenom. 52 (1990) 837.

[21] Bouchet D., Colliex C., Flora P., KrivaneK O.L., Mory C., TenCÉ M., Microsc. Microanal Microstruct. 1 (1990) 443.

[22] SAWATZKY G.A., Ultramicroscopy 34 (1990) Guest editorial for $\mathrm{n}^{\circ} 4$.

[23] SAWATZKY G.A., Microsc. Microanal. Microstruct. 2 (1991) 153.

[24] BRYDSON R., SAUER H., ENGEL W., ZEITLER E., Microsc. Microanal Microstruct. 2 (1991) 159.

[25] REZ P., WENG X.D., MA H., Microsc. Microanal. Microstruct. 2 (1991) 143.

[26] Brydson R., Sauer H., Engel W., ThOmas J.M., Zeitler E., J. Chem. Soc. Chem. Commun. 15 (1989) 1010.

[27] Brydson R., SAuer H., Engel W., Thomas J.M., Zeitler E., Kosugi N., Kuroda H. J. Phys.: Condens. Matter 1 (1989) 797.

[28] Rowley P.N., Brydson R., Little J., Sauders R.J., Philos. Mag. B62 (1990) 229.

[29] Pearson D.H., Fultz B., Ahn C.C., Appl. Phys. Lett. 53 (1988) 1405.

[30] KRIShNAN K.M., MENON E.S.K., HUANG P., Singh P.P., de FonTAINE D., to be published (1991).

[31] Morrison T.I., Brodsky M.B., ZaluZec N.J., Sill L.R., Phys. Rev. B32 (1987) 3107.

[32] Morrison T.I., Foiles C.L., Pease D.M., Zaluzec N.J., Phys. Rev. B32 (1987) 3739.

[33] Kurata H., TANAKa N., Microsc. Microanal. Microstruct. 2 (1991) 183.

[34] HOWIE A., WALSH C.A., Microsc. Microanal. Microstruct. 2 (1991) 171. 